\title{
Study of moderate to severe hyponatremia in patients admitted to medical intensive care units (MICU) in a tertiary care hospital
}

\author{
Dnyaneshwar Malharrao Ghuge ${ }^{1^{*}}$, Balasaheb Eknathrao Karad ${ }^{2}$
}

${ }^{1}$ Associate Professor, ${ }^{2}$ Professor, Department of Medicine, Pacific Institute of Medical Sciences, Udaipur, Rajasthan, INDIA. Email: drdmghuge@yahoo.com, karadbalasaheb@gmail.com

Abstract Background: Hyponatremia is one of the most common electrolyte disturbances, occurring in approximately $14 \%-42 \%$ of hospitalized patients, and it is associated with higher mortality. Appropriate management of hyponatremia is often challenging due to both numerous pathophysiological mechanisms and multiple underlying pathological conditions. ${ }^{8}$ In present article we aimed to study etio-pathogenesis of moderate to severe hyponatremia in patients admitted to MICU in a tertiary care hospital. Material and Methods: Present study was an observational study conducted in the ICU of department of general medicine in patients more than 18 yrs age, with documented hyponatremia ( serum sodium concentration less than $135 \mathrm{meq} / \mathrm{L}$ ). Data was collected in a proforma, data entered in Microsoft excel sheet. Statistical analysis was done using descriptive statistics. Results: During study period, 72 patients with hyponatremia were included in present study. Most common age group was more than 70 years age and 40-49 years $(21 \%)$ followed by $50-59$ years $(19 \%)$. Male $(63 \%)$ patients were more than female $(38 \%)$ patients. Patients with hyponatremia were classified based on serum sodium levels into mild (130-134 mEq/L), moderate $(125-129 \mathrm{mEq} / \mathrm{L})$, and severe $(<125 \mathrm{mEq} / \mathrm{L})$. Mild, moderate and severe hyponatremia was noted $47 \%, 32 \%$ and $21 \%$ patients. In present study, most common etiology of hyponatremia was GI fluid loss (AGE, vomiting) (22\%), cerebrovascular accident (13\%), chronic liver disease (13\%), malignancy (11\%) and renal failure (10\%). Clinically patients were distributed as euvolemic (most common -64\%), hypervolemic (13\%) or hypovolemic (24\%). Patients were diagnosed to have syndrome of inappropriate antidiuretic hormone secretion (SIADH) if they satisfied the Bartter and Schwartz criteria. In present study we noted 13\% incidence SIADH. Patients responded well to standard treatment. $10 \%$ mortality was noted. There were no cases of central pontine myelinolysis Conclusion: Euvolumic, mild, asymptomatic hyponatremia is common in medical intensive care units.

Keywords: electrolyte disturbance, hyponatremia, serum sodium, medical intensive care unit

\section{*Address for Correspondence:}

Dr Dnyaneshwar Malharrao Ghuge, Associate Professor, Department of Medicine, Pacific Institute of Medical Sciences, Udaipur, Rajasthan, INDIA.

Email: karadbalasaheb@gmail.com

Received Date: 09/01/2020 Revised Date: 02/02/2020 Accepted Date: 24/02/2020

DOI: https://doi.org/10.26611/10211636

This work is licensed under a Creative Commons Attribution-NonCommercial 4.0 International License. (cc)) EY-NC

\begin{tabular}{|l|l|}
\hline \multicolumn{2}{|c|}{ Access this article online } \\
\hline Quick Response Code: & Website: \\
\hline & www.medpulse.in \\
& \\
\hline
\end{tabular}

\section{INTRODUCTION}

Sodium is the most prevalent cation in the extracellular fluid. Under normal circumstances serum sodium levels are maintained within a tight physiological range of between $135-145 \mathrm{mmol} / 1$. Despite great variation in the intake of both sodium and water, close control of serum sodium is maintained via control of the excretion of water and sodium. ${ }^{1}$ Hyponatremia is one of the most common electrolyte disturbances, occurring in approximately $14 \%-$ $42 \%$ of hospitalized patients, and it is associated with higher mortality. ${ }^{2,3}$ Even mild hyponatremia has been associated with increased morbidity, including gait disturbances, cognitive impairment, osteoporosis, falls and fractures, and mortality. ${ }^{4}$ Hyponatraemia has a wide clinical spectrum varying from absence of symptoms to seizures, coma and death. Nausea and malaise are the earliest findings and may be seen when the plasma sodium concentration falls below 125 to $130 \mathrm{mmol} / \mathrm{l}$. This may be followed by headache, lethargy, obtundation, and eventually seizure, coma and respiratory arrest if the plasma sodium concentration falls below 115 to 120 $\mathrm{mmol} / \mathrm{l}$. Serum sodium concentration and plasma osmolarity usually maintained under homeostatic 
mechanisms i.e. thirst, antidiuretic hormone (ADH), and kidney function. ${ }^{5,6}$ It is unclear if hyponatremia is a marker for poor prognostic outcomes or merely a reflection of disease severity. Its presence suggests a worse prognosis in patients with liver cirrhosis, pulmonary hypertension, myocardial infarction, chronic kidney disease, hip fractures, and pulmonary embolism. ${ }^{7}$ Appropriate management of hyponatremia is often challenging due to both numerous pathophysiological mechanisms and multiple underlying pathological conditions. ${ }^{8}$ In present article we aimed to study moderate to severe hyponatremia in patients admitted to MICU in a tertiary care hospital.

\section{MATERIAL AND METHODS}

Present study was an observational study conducted in the ICU of Department of Medicine, Pacific Institute of Medical Sciences, Udaipur. Study duration was of 1 year and ethical committee approval was taken.

All ICU admitted patients undergo serum electrolytes (serum sodium) estimation.

\section{Inclusion Criteria:}

- Patients more than 18 yrs. age, admitted in medicine ICU's with documented hyponatremia ( serum sodium concentration less than $135 \mathrm{meq} / \mathrm{L}$ ) Exclusion Criteria:

- Patients with pseudohyponatremia (hyponatremia in the absence of any obvious etiology and presence of hyperproteinemia and/or hypertriglyceridemia)

- Patients with hyperglycemia, hyperlipidemia, paraproteinemias (known cases), as well as those receiving mannitol.

Informed consent for participation in present study was taken from patients. Demographic data, detailed history (history of symptoms of hyponatremia, predisposing factors and pre- existing illnesses, presence of altered sensorium, postural dizziness, lethargy and seizures, history of illnesses causing hyponatremia, history of fluid loss as in vomiting, diarrhea, diuretic use) was taken in all patients. Detailed clinical evaluation for hydration status, signs of hypovolemia ( tachycardia, orthostatic falls in blood pressure, decreased skin turgor, dry mucous membranes and decreased peripheral perfusion with a delayed capillary refill more than three seconds), hypervolemia signs ( presence of anasarca, ascites, symmetrical and pitting pedal edema and raised jugular venous pressure), CNS examination was done to document the signs of raised ICP (bradycardia, hypertension and papilloedema) was done and findings noted. Accordingly patients were divided into hypervolemic, hypovolemic and euvolemic states. CNS examination was repeated after the correction of hyponatremia. Complete blood count $(\mathrm{Hb}$, TLC, DLC and platelet count), Urine routine examination and microscopic examination, serum sodium (baseline, every alternate day in symptomatic patients, daily in symptomatic patients, 6-8 hourly in patients with severe hyponatremia on $3 \%$ saline infusion). serum blood urea nitrogen (BUN) and glucose levels (for calculation of serum osmolality). Serum sodium estimation was done in the "Easylite" Automated Analyzer by Ion Selective Electrode (ISE) technology. Thyroid function test (TFT), serum cortisol levels, urine spot sodium, urine urea, urine creatinine, urine uric acid, and imaging studies were done as required. Data was collected in a proforma, data entered in Microsoft excel sheet. Statistical analysis was done using descriptive statistics.

\section{RESULTS}

During study period, 72 patients with hyponatremia were included in present study. Most common age group was more than 70 years age and 40-49 years (21\%) followed by $50-59$ years $(19 \%)$. Male $(63 \%)$ patients were more than female $(38 \%)$ patients.

Table 1: Age and gender distribution

\begin{tabular}{cccc}
\hline AGE (yrs.) & Male & Female & Total \\
\hline $19-29$ & $4(6 \%)$ & $2(3 \%)$ & $36(8 \%)$ \\
$30-39$ & $6(8 \%)$ & $3(4 \%)$ & $9(13 \%)$ \\
$40-49$ & $8(11 \%)$ & $7(10 \%)$ & $15(21 \%)$ \\
$50-59$ & $10(14 \%)$ & $4(6 \%)$ & $14(19 \%)$ \\
$60-69$ & $8(11 \%)$ & $5(7 \%)$ & $13(18 \%)$ \\
$\geq 70$ & $9(13 \%)$ & $6(8 \%)$ & $15(21 \%)$ \\
\hline Total & $45(63 \%)$ & $27(38 \%)$ & 72 \\
\hline
\end{tabular}

Patients with hyponatremia were classified based on serum sodium levels into mild (130-134 mEq/L), moderate (125$129 \mathrm{mEq} / \mathrm{L})$, and severe $(<125 \mathrm{mEq} / \mathrm{L})$. Mild, moderate and severe hyponatremia was noted $47 \%, 32 \%$ and $21 \%$ patients.

Table 2: Distribution according to severity of hyponatremia

\begin{tabular}{cccc}
\hline SEVERITY & Male & Female & Total \\
\hline Mild & $22(31 \%)$ & $12(17 \%)$ & $34(47 \%)$ \\
Moderate & $14(19 \%)$ & $9(13 \%)$ & $23(32 \%)$ \\
Severe & $9(13 \%)$ & $6(8 \%)$ & $15(21 \%)$ \\
\hline
\end{tabular}

In present study, most common etiology of hyponatremia was GI fluid loss (AGE, vomiting) (22\%), cerebrovascular accident (13\%), chronic liver disease (13\%), malignancy $(11 \%)$ and renal failure $(10 \%)$.

Table 3: Etiology of hyponatremia

\begin{tabular}{ccc}
\hline Etiology & $\begin{array}{c}\text { No. of } \\
\text { patients }\end{array}$ & Percentage \\
\hline Chronic liver disease/ Liver cirrhosis & 16 & $22 \%$ \\
Cerebrovascular accident & 9 & $13 \%$ \\
Gl fluid loss (AGE, vomiting) & 9 & $13 \%$ \\
Malignancy & 8 & $11 \%$ \\
Renal failure & 7 & $10 \%$ \\
Congestive Cardiac Failure & 7 & $10 \%$ \\
Lung abscess & 6 & $8 \%$ \\
Multi-factorial & 6 & $8 \%$ \\
Nephrotic syndrome & 4 & $6 \%$ \\
\hline
\end{tabular}


Clinically patients were distributed as euvolemic (most common $-64 \%)$, hypervolemic $(13 \%)$ or hypovolemic $(24 \%)$.

Table 4: Type of hyponatraemia.

\begin{tabular}{ccccc}
\hline Aetiology & Mild & Moderate & Severe & Total \\
\hline Hypovolaemi & $7(10 \%)$ & $5(7 \%)$ & $5(7 \%)$ & $17(24 \%)$ \\
c & & & & \\
Hypervolemic & $4(6 \%)$ & $3(4 \%)$ & $2(3 \%)$ & $9(13 \%)$ \\
Euvolemic & $23(32 \%)$ & $15(21 \%)$ & $8(11 \%)$ & $46(64 \%)$ \\
& $34(47 \%)$ & $23(32 \%)$ & $15(21 \%)$ & 72 \\
\hline
\end{tabular}

Patients were diagnosed to have syndrome of inappropriate antidiuretic hormone secretion (SIADH) if they satisfied the Bartter and Schwartz criteria. In present study we noted $13 \%$ incidence SIADH. Patients responded well to standard treatment. $10 \%$ mortality was noted. There were no cases of central pontine myelinolysis

\section{DISCUSSION}

The renal function, sensation of thirst, concentrating abilities and hormonal regulation of water and solutes are usually impaired in hospitalized patients which makes such individuals susceptible to alterations in serum sodium and water. ${ }^{9}$ Various pathophysiological factors, such as nutritional status, gastrointestinal absorption capacity, coexistent acid-base abnormalities, pharmacological agents, other co-morbid diseases (mainly renal disease) or acute illness, alone or in combination, play a key role. ${ }^{10}$ Electrolyte disorders are common in clinical practice and mainly encountered in hospital populations occurring in a broad spectrum of patients (from asymptomatic to critically ill) and being associated with increased morbidity and mortality. ${ }^{11}$ Hyponatremia may cause complications in critically ill patients, including neurologic deficits, muscle weakness and cramps, hyperventilation, impairment in gluconeogenesis, and decreased left ventricular function. ${ }^{12}$ In a huge multicentric trial with 151,486 patients, it was demonstrated that all types and grades of dysnitremias were associated with increased risk adjusted and raw hospital mortality rates. The odds ratios for mild, moderate, and severe hyponatremias were $1.32,1.89$, and 1.81, respectively. ${ }^{13}$ A Dutch systematic review of 53 studies showed that the prevalence of mild hyponatremia was $22.2 \%$ in geriatric hospital wards, $6.0 \%$ in nongeriatric wards, and $17.2 \%$ in the intensive care unit. ${ }^{14}$ Studies suggest that hyponatremia may be present in $15 \%-30 \%$ of patients in chronic care facilities. ${ }^{15}$ Chatterjee et al.. studied the incidence and clinical profile of hyponatremia in medically ill patients and found the incidence to be $16.4 \%{ }^{16}$ Mittal et al. reported the incidence of hyponatremia to be $4 \%$ per cent in hospitalised patients. ${ }^{17}$ Most common age group was more than 70 years in present study. The incidence is much more in the elderly mainly owing to the impaired ability to maintain water and electrolyte homeostasis in response to dietary and environmental changes. Babaliche et al. ${ }^{18}$ reported male predominance $(59 \%)$ in the incidence of hyponatremia. A similar gender distribution pattern was reported by Rahil et al.., ${ }^{19}$ wherein $33(62.3 \%)$ patients with hyponatremia were males and 20 (37.7\%) were females. Similar findings were noted in present study. Nandani Chatterjee et al.., ${ }^{15}$ conducted a study and noted that, most common underlying predisposing factor for hyponatremia was gastro-intestinal fluid loss followed by cerebrovascular accidents and pulmonary sepsis. Euvolemic hyponatremia comprises the bulk of the patients (50.74\%). A significant number suffer from SIADH (32.84\%). A large fraction of cases $(48.21 \%)$ are asymptomatic. In study by Baji $\mathrm{P}$ et al.. ${ }^{20} 34 \%$ had euvolemic hyponatremia, 19\% had hypervolemic hyponatremia associated with oedematous disorders and $17 \%$ had hypovolemic conditions, chiefly related to GI fluid loss or diuretic use. Similar findings were noted in present study. Bissram et al... ${ }^{21}$ found that symptomatic hyponatremia was associated with volume depletion (32.6\%), congestive heart failure (HF) $(26 \%)$, SIADH (26\%), thiazide diuretic use (26\%) and selective serotonin re-uptake inhibitor use (26\%). In $21.7 \%$ of cases, the cause was multifactorial (congestive HF, SIADH or medication use with volume depletion). Hyponatremia a frequent complication of advanced cirrhosis, is related to an impairment in the renal capacity to eliminate solute-free water that causes a retention of water that is disproportionate to the retention of sodium, thus causing a reduction in serum sodium concentration and hypoosmolality. ${ }^{22}$ Hyponatremia is not a homogenous disorder and depending upon the volume status of the patient can either be euvolemic, hypervolemic or hypovolemic. Each etiology and type of hyponatremia requires a different approach to treatment. ${ }^{23}$ Euvolemic hyponatremia was most common in present study. Similar findings were noted by Nandini Chatterjee et al.., ${ }^{15}$ Mahim Mittal et al. ${ }^{16}$ Syndrome of inappropriate antidiuresis (SIAD) is widely assumed the commonest cause of euvolemic hyponatremia, but some time it may be over diagnosed particularly in dehydrated elderly patients. Due to incorrect diagnosis these patients may be mismanaged because the management of the euvolemic hyponatremia (SIAD) is exact opposite to the management of hypovolemic hyponatremia. ${ }^{24,25}$ Hyponatremic encephalopathy is the most serious complication of hyponatremia. Significant risk factors for developing hyponatremic encephalopathy include female sex, hypoxia, and underlying central nervous system disease. The symptoms of hyponatremic encephalopathy are largely related to cerebral edema. Hyponatremic encephalopathy constitutes a medical emergency because it might lead to death or permanent neurologic deterioration due to transtentorial herniation or 
respiratory arrest if untreated. ${ }^{26,27}$ The plethora of underlying etiologies warrants a careful differential diagnosis, which should take into consideration comorbidities, concurrent medication, findings from the clinical examination and laboratory measurements, which in turn will guide management decisions in cases of suspected or diagnosed hyponatremia.

\section{CONCLUSION}

Euvolemic, mild, asymptomatic hyponatremia is common in medical intensive care units. A systematic approach to the diagnosis of hyponatremia with the application of simple diagnostic algorithms using history, clinical examination, and laboratory findings to establish the mechanism of hyponatremia can significantly improve the assessment and management of hyponatremia.

\section{REFERENCES}

1. Houillier P. Sodium homeostasis. Nephrol Ther 2007; 3 Suppl 2: S91-S93.

2. Waikar SS, Mount DB, Curhan GC: Mortality after hospitalization with mild, moderate, and severe hyponatremia. Am J Med 122: 857-865, 2009

3. Upadhyay A, Jaber BL, Madias NE: Incidence and prevalence of hyponatremia. Am J Med 119 [Suppl 1]: S30-S35, 2006

4. Liamis G, Rodenburg EM, Hofman A, Zietse R, Stricker $\mathrm{BH}$, Hoorn EJ. Electrolyte disorders in community subjects: prevalence and risk factors. Am J Med. 2013 Mar; 126(3): 256-63.

5. Verbalis JG, Goldsmith SR, Greenberg A, Korzelius C, Schrier RW, Sterns RH, et al. Diagnosis, evaluation, and treatment of hyponatremia: expert panel recommendations. Am J Med. 2013 Oct;126(10 Suppl 1):S1-42.

6. Sterns RH, Hix JK, Silver SM. Management of hyponatremia in the ICU. Chest. 2013 Aug;144(2):672-9

7. Hoorn EJ, et al. Hyponatremia and mortality: moving beyond associations. Am J Kidney Dis. 2013;62(1):139149.

8. Bennani S-L, Abouqal R, Zeggwagh A-A, Madani N, Abidi K, Zekraoui A et al.. Incidence, causes and prognostic factors of hyponatremia in intensive care. Rev Med Interne 2003; 24: 224-229.

9. Braun MM, Barstow CH2, Pyzocha NJ. Diagnosis and management of sodium disorders: hyponatremia and hypernatremia. Am Fam Physician. 2015 Mar 1;91(5):299-307.

10. Liamis G, Liberopoulos E, Barkas F, Elisaf M. Diabetes mellitus and electrolyte disorders. World Journal of Clinical Cases: WJCC. 2014; 2(10):488

11. Kughapriya $P$, Evangeline J. Evaluation of serum electrolytes in Ischemic Heart Disease patients. National Journal of Basic Medical Sciences. 2016; 6(4):1-14.
12. Verbalis JG, Goldsmith SR, Greenberg A, et al.. Diagnosis, evaluation, and treatment of hyponatremia: expert panel recommendations. Am J Med 2013;126:S1S42.

13. Funk GC, Lindner G, Druml W, Metnitz B, Swarz C, Bauer $\mathrm{P}$, et al.. Incidence and prognosis of dysnatremias present on ICU admission. Intensive Care Med 2010;36:304-11.

14. Mannesse CK, et al.. Prevalence of hyponatremia on geriatric wards compared to other settings over four decades: a systematic review. Ageing Res Rev. 2013;12(1):165-173.

15. Janicic N, Verbalis JG. Evaluation and management of hypoosmolality in hospitalized patients. Endocrinol Metab Clin North Am 2003;32:459-81.

16. Chatterjee N, Sengupta N, Das C et al.. A descriptive study of hyponatraemia in a tertiary care hospital of Eastern India. Indian J Endocrinol Metab 2012; 16: 288-91.

17. Mittal M, Deepshikha, Khurana H. Profile of hyponatraemia in a tertiary care centre in North India. Int J Adv Med 2016; 3: 1011-5.

18. Babaliche P, Madnani S, Kamat S. Clinical profile of patients admitted with hyponatremia in the medical intensive care unit. J Crit Care Med 2017;21:819-24.

19. Rahil A, Khan F, Al Badri M. Clinical profile of hyponatraemia in adult patients admitted to Hamad General Hospital, Qatar: Experience with 53 cases. J Clin Diagn Res 2009;3:1419-25.

20. Baji P, Borkar S. Clinico-etiological profile and outcome of hyponatremia in hospitalised adult patients. Int J Sci Rep 2015;1:293-8.

21. Bissram M, Scott FD, Liu L, Rosner MH. Risk factors for symptomatic hyponatraemia: The role of pre-existing asymptomatic hyponatraemia. Intern Med J 2007;37:14955 .

22. Ginès $P$ and Guevara $M$, Hyponatremia in cirrhosis: pathogenesis, clinical significance, and management. Hepatology; 2008, 48:1002

23. Mount DB. Fluid and electrolyte disorders. In: Kasper DL, Hauser SL, Jamerson JL, Fauci AS, Longo DL, Loscalzo J, eds. 19th ed. Harrison's Principles of Internal Medicine. New York: McGraw-Hill Education, 2015; pp. 298-9.

24. Soiza RL, Talbot HS. Management of hyponatremia in older people: Old threats and new opportunities. Ther Adv Drug Saf 2011;2:9-17.

25. Ayus JC, Negri AL, Kalantar-Zadch K, Moritz ML. Is chronic hyponatremia a novel risk factor for hip fracture in the elderly? Nephrol Dial Transplant 2012;27:3275-731.

26. Moritz ML, Ayus JC. New aspects in the pathogenesis, prevention, and treatment of hyponatremic encephalopathy in children. Pediatr Nephrol. 2010;25(7):1225-1238.

27. Spasovski G, Vanholder R, Allolio B, et al.. Clinical practice guideline on diagnosis and treatment of hyponatraemia. Nephrol Dial Transplant. 2014;29 Suppl 2:i1-i39.

\section{Source of Support: None Declared}

Conflict of Interest: None Declared 